\title{
On the accuracy of retrieved wind information from Doppler lidar observations
}

\author{
By F. DAVIES ${ }^{1 *}$, C. G. COLLIER ${ }^{1}$, K. E. BOZIER ${ }^{1}$ and G. N. PEARSON ${ }^{2}$ \\ ${ }^{1}$ University of Salford, $U K$ \\ ${ }^{2}$ Qineti $Q \dagger, U K$
}

(Received 20 July 2001; revised 30 August 2002)

\begin{abstract}
SUMMARY
A single pulsed Doppler lidar was successfully deployed to measure air flow and turbulence over the Malvern hills, Worcester, UK. The DERA Malvern lidar used was a $\mathrm{CO}_{2} 10.6 \mu \mathrm{m}$ pulsed Doppler lidar. The lidar pulse repetition rate was $120 \mathrm{~Hz}$ and had a pulse duration of $0.6 \mu \mathrm{s}$. The system was set up to have 41 range gates with range resolution of $112 \mathrm{~m}$. This gave a theoretical maximum range of approximately $4.6 \mathrm{~km}$. The lidar site was $2 \mathrm{~km}$ east of the Malvern hill ridge which runs in a north-south direction and is approximately $6 \mathrm{~km}$ long. The maximum height of the ridge is $430 \mathrm{~m}$. Two elevation scans (Range-Height Indicators) were carried out parallel and perpendicular to the mean surface flow. Since the surface wind was primarily westerly the scans were carried out perpendicular and parallel to the ridge of the Malvern hills.

The data were analysed and horizontal winds, vertical winds and turbulent fluxes were calculated for profiles throughout the boundary layer. As an aid to evaluating the errors associated with the derivation of velocity and turbulence profiles, data from a simple idealized profile was also analysed using the same method. The error analysis shows that wind velocity profiles can be derived to an accuracy of $0.24 \mathrm{~m} \mathrm{~s}^{-1}$ in the horizontal and $0.3 \mathrm{~m} \mathrm{~s}^{-1}$ in the vertical up to a height of $2500 \mathrm{~m}$. The potential for lidars to make turbulence measurements, over a wide area, through the whole depth of the planetary boundary layer and over durations from seconds to hours is discussed.
\end{abstract}

KEYWORDS: Atmospheric measurements Boundary layer

\section{INTRODUCTION}

Measurement of meteorological variables in the atmospheric boundary layer has been largely implemented with in situ sensors mounted on towers, airplanes and balloons. Such instruments are limited to point or line measurements. In more recent years remote-sensing instrumentation such as radar, lidar and sodar, which scan large areas over much shorter time-scales, have been developed. Lidars in particular are useful for measurement of mean wind and turbulence parameters over extended areas in the atmospheric boundary layer. The advantage is that they have extremely small beam side lodes, thus eliminating problems due to ground echoes. Kunkel et al. (1980) carried out a direct comparison between point anemometer readings and derived lidar measurements using a $0.694 \mu \mathrm{m}$ laser looking at the cross-correlation of moving aerosol inhomogeneities.

The first reported use of a Doppler lidar for vertical profiles of wind and turbulence measurements was made by Eberhard et al. (1989). The basis of Doppler lidar is reflection of light back from scattered aerosol particles which are used as tracers of the air motion. This backscattered light gives a measure of the radial component of the wind velocity. Using a $360^{\circ}$ azimuth scan (or Plan Position Indicator (PPI)) radial wind velocities were analysed using a Doppler radar technique known as Vertical Azimuth Display or VAD (Browning and Wexler (1968)) to produce horizontal and vertical wind profiles. Turbulence profiles were then calculated using an application of the VAD technique demonstrated by Wilson (1970).

In this paper we follow an alternative approach outlined and tested by Gal-Chen et al. (1992) (hereafter G-C92). Again this is a procedure for deriving vertical profiles of horizontal and vertical winds from a single Doppler lidar. The lidar which was operated

\footnotetext{
* Corresponding author: School of Environment and Life Sciences, Peel Building, University of Salford, Greater Manchester, UK. e.mail: F.Davies@ Salford.ac.uk

$\dagger$ Formerly the Defence Evaluation and Research Agency.

(C) Royal Meteorological Society, 2003.
} 
by the National Oceanic and Atmospheric Administration (NOAA) Wave Propagation Laboratory (WPL) was a $\mathrm{CO}_{2}(10.6 \mu \mathrm{m})$ laser, had a range resolution of $150 \mathrm{~m}$ and a pulse repetition rate of $20 \mathrm{~Hz}$. The lidar could scan from $0^{\circ}$ up to $42^{\circ}$ in elevation and also vertically.

The design of the experiment by G-C92 was a compromise between two basic requirements; the need to scan every resolved volume in the boundary layer and the requirement that it should be done fast enough that the assumption of a statistical steady state is valid. The method that G-C92 chose, details of which are given later, was to perform two elevation scans (or Range-Height Indicators, RHIs). The first scan was in the direction of the mean surface wind (defined to be the $x-z$ plane) and the second scan was in a direction orthogonal to this (in the $y-z$ plane). The two alternate scans together took approximately 72 seconds and measurements were taken continuously for a 1-hour period. These scans were filtered to remove erroneous data and then averaged so stable statistics could be obtained.

Their results show a wind profile that is constant up to a height of $500 \mathrm{~m}$, with a southerly wind at the surface turning slightly westerly with height. A maximum in the wind speed of $17 \mathrm{~m} \mathrm{~s}^{-1}$ is seen at approximately $1 \mathrm{~km}$. Temperature soundings throughout the duration of the experiment show an initial mixed-layer height of $700 \mathrm{~m}$, growing to $1300 \mathrm{~m} 3$ hours later. The vertical velocity is negative $\left(-0.6 \mathrm{~m} \mathrm{~s}^{-1}\right.$ at $\left.600 \mathrm{~m}\right)$ throughout the boundary layer and is shown to decrease with height. The momentum flux, $\overline{u^{\prime} w^{\prime}}$, is shown to be approximately $-0.5 \mathrm{~m}^{2} \mathrm{~s}^{-2}$ throughout the mixed layer and to have a maximum of $-2.5 \mathrm{~m}^{2} \mathrm{~s}^{-2}$ between 1.2 and $1.3 \mathrm{~km}$. They note that the large momentum fluxes at this height may be due to interactions between convection in the mixed layer and waves in a stable layer. This zone of interaction is termed the 'critical layer' and is discussed by Kuettner et al. (1987).

The Defence Evaluation and Research Agency (DERA) Malvern lidar is a $\mathrm{CO}_{2}$ (10.6 $\mu \mathrm{m})$ pulsed Doppler lidar (Pearson and Collier 1999). With the pulse repetition rate set at $120 \mathrm{~Hz}$ and a pulse duration of $0.6 \mu \mathrm{s}$ the system is set up to have 41 range gates with range resolution of $112 \mathrm{~m}$. This gives a theoretical maximum range of approximately $4.6 \mathrm{~km}$. The actual maximum range depends on the concentrations of aerosol particles in the atmosphere. There is a minimum range of $560 \mathrm{~m}$ below which velocity estimates are not reliable. The scanning capabilities of the DERA lidar are the full $360^{\circ}$ for a PPI scan and $180^{\circ}$ for an RHI scan. The scan rate during this experiment was set at $1^{\circ}$ per second.

\section{MODELLING RADIAL WINDS}

(a) Retrieving wind measurements

G-C92 described a method of deriving wind profiles from elevation scans (RHI) of the lidar. In their experiment two scans are carried out at right angles which retrieve data in the $(x-z)$ and $(y-z)$ planes. In this paper we only examine the along wind component, $(x-z)$. The radial wind denoted by $\alpha$ can be decomposed into the $u$ and $w$ components as shown:

$$
\begin{aligned}
& \alpha_{+}=u \cos \Theta+w \sin \Theta \quad \text { with the lidar pointing in the }+x \text { direction } \\
& \alpha_{-}=-u \cos \Theta+w \sin \Theta \quad \text { with the lidar pointing in the }-x \text { direction }
\end{aligned}
$$

where $\Theta$ is the elevation angle of the lidar beam. The data were then separated into height bins, as shown in Fig. 1. A height-bin resolution of $50 \mathrm{~m}$ was used and the separate height bins, $z$, are denoted by index $i$. If assumptions are made about the 


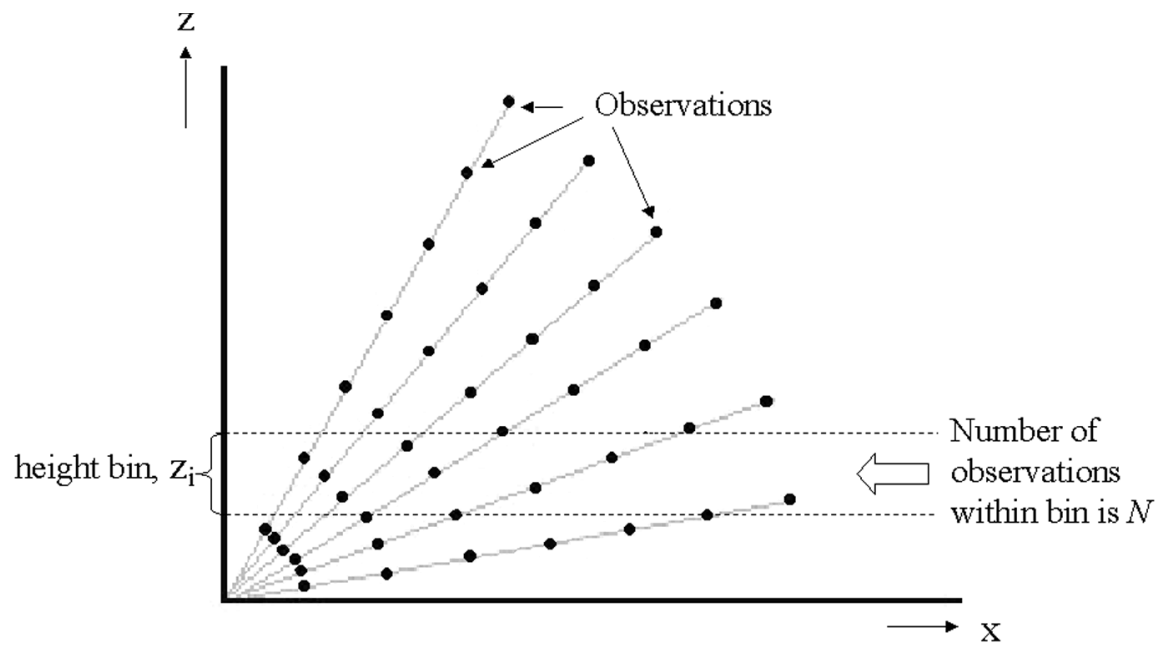

Figure 1. The partitioning of the lidar data points into height bins.

turbulence being horizontally homogeneous and the observations being unbiased, the ensemble average of the velocities in each bin can then be denoted by $\bar{u}_{i}$ and $\bar{w}_{i}$ and the equations can be rewritten

$$
\alpha_{i j \pm}= \pm \bar{u}_{i} \cos \Theta_{j}+\bar{w}_{i} \sin \Theta_{j} \quad j=1,2, \ldots, N_{i}
$$

in the along wind direction where $N_{i}$ is the number of observations in bin $z_{i}$ in the along wind direction. This creates $\bar{u}$ and $\bar{w}$ profiles which are effectively spatially averaged over the coverage area of the lidar.

For height bin $z_{i}$, Eq. (3) then defines $2 N_{i}$ equations with two unknowns $\left(\bar{u}_{i}, \bar{w}_{i}\right)$ which can be solved using a least-square-fit technique.

G-C92 then describe a similar technique to calculate the turbulence parameters. Initially the variables are separated to their mean and perturbation components:

$$
u=\bar{u}_{i}+u^{\prime} ; \quad w=\bar{w}_{i}+w^{\prime} ; \quad \alpha=\bar{\alpha}_{i}+\alpha^{\prime} .
$$

The mean radial velocity is then defined as

$$
\bar{\alpha}_{i \pm}= \pm \bar{u}_{i} \cos \Theta+\bar{w}_{i} \sin \Theta
$$

in the along wind direction.

Assuming horizontal homogeneity for a bin corresponding to a height $z_{i}$ the perturbation radial velocity, $\alpha^{\prime}$, becomes

$$
\left(\alpha^{\prime 2}\right)_{i j}= \pm \overline{u^{\prime} w_{i}^{\prime}} \sin 2 \Theta_{j}+\overline{w_{i}^{\prime 2}} \sin ^{2} \Theta_{j}+\overline{u_{i}^{\prime 2}} \cos ^{2} \Theta_{j} \quad j=1,2, \ldots, N_{i}
$$

in the along wind direction, where $\Theta$ is again the lidar beam elevation angle.

The second moments $\overline{u_{i}^{\prime 2}}, \overline{w_{i}^{\prime 2}}$ and $\overline{u^{\prime} w_{i}^{\prime}}$ can be obtained using a similar least-square method for the $2 N_{i}$ equations.

\section{(b) Modelling radial winds}

As described above, the DERA lidar retrieves data at an angular resolution of $1^{\circ}$ and for a RHI scans the entire $180^{\circ}$. To simulate this data set-up an idealized dataset 


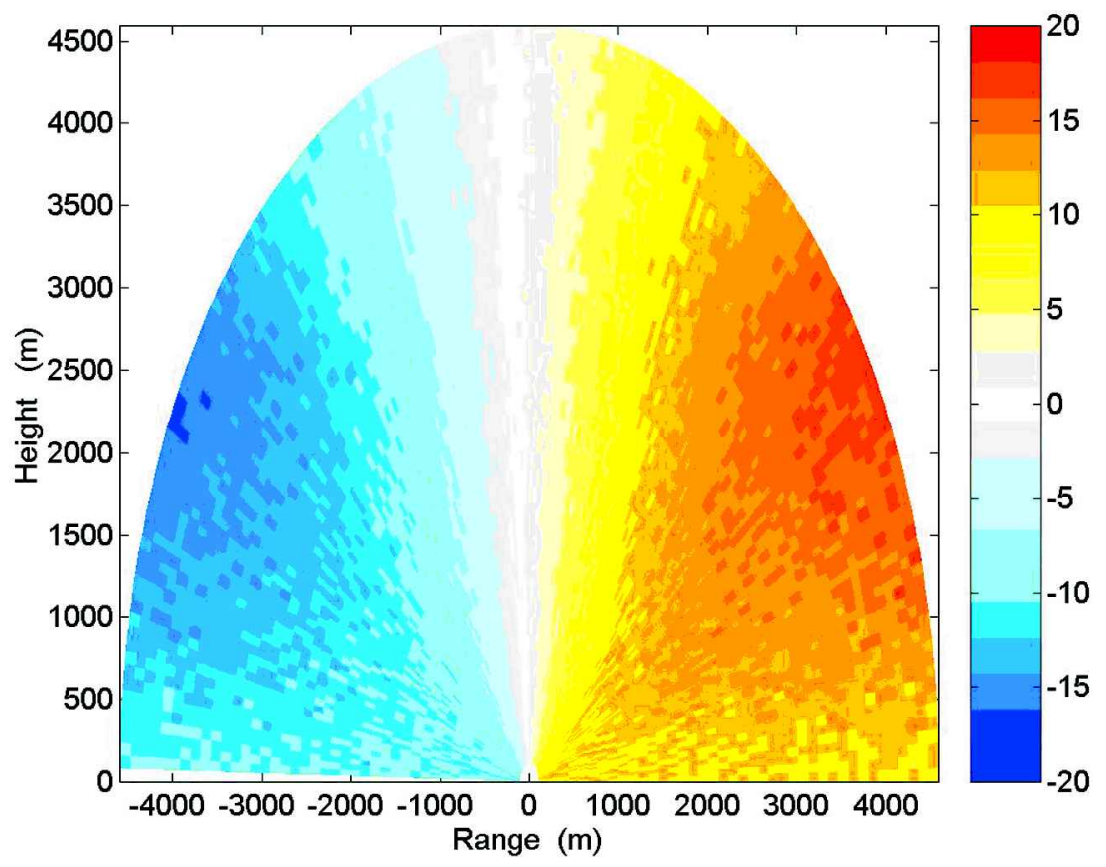

Figure 2. Idealized radial wind velocity scan. (Colour bar shows wind velocity in $\mathrm{m} \mathrm{s}^{-1}$.)

was created by calculating radial winds in the $(x-z)$ plane from a defined wind velocity profile. The $U$-component of the mean wind was defined to be $10 \mathrm{~m} \mathrm{~s}^{-1}$ at the ground increasing linearly to $20 \mathrm{~m} \mathrm{~s}^{-1}$ at $2500 \mathrm{~m}$, and constant above $2500 \mathrm{~m}$. The vertical component of the mean wind was defined to be a constant $1 \mathrm{~m} \mathrm{~s}^{-1}$ at all heights. To simulate the turbulence, perturbations were added to the mean wind components. The perturbations were calculated using a random number generator with zero mean and standard deviations defined by $\sigma_{u}=0.72$ and $\sigma_{w}=0.37$. The friction velocity, which is related to the surface Reynolds stress (Panofsky and Dutton 1984), was then set to $u^{*}=0.09$. This gave the ratio of standard deviation of velocity to friction velocity as:

$$
\begin{aligned}
\sigma_{u} u^{*} & =2.39 \\
\sigma_{w} u^{*} & =1.25 .
\end{aligned}
$$

These ratio values are given by Panofsky and Dutton (1984) as average values in the atmospheric surface layer for near-neutral conditions over flat terrain. The amount of turbulence was kept constant with height in the 'idealized' atmosphere to give an indication of how the errors change with height due to purely the configuration of the scan. The amount of turbulence was set to reflect a true atmospheric radial velocity scan.

Figure 2 shows the resulting idealized radial velocity scan. The figure shows wind flowing from the left to the right of the plot. The data were processed as described above using information from $0^{\circ}$ to $60^{\circ}$ in elevation. Above $60^{\circ}$ in elevation the errors associated with the decomposition of $u$ and $w$ from the radial wind $\alpha$ are very large. Range gates 6-41 were used as these are the ones that are available in the real data. Horizontal and vertical wind profiles were derived for heights up to $3500 \mathrm{~m}$ and are shown in Figs. 3 and 4 compared with defined mean wind components. 


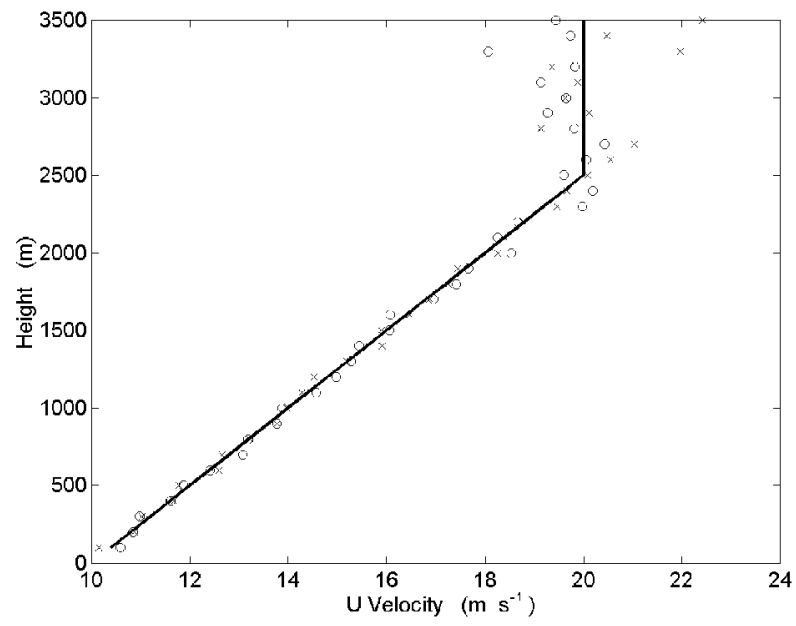

Figure 3. The line shows the mean $u$-wind profile input into the idealized model; crosses $(\times)$ and circles (०) denote horizontal wind velocities, derived from the idealized model, from positive and negative range data respectively.

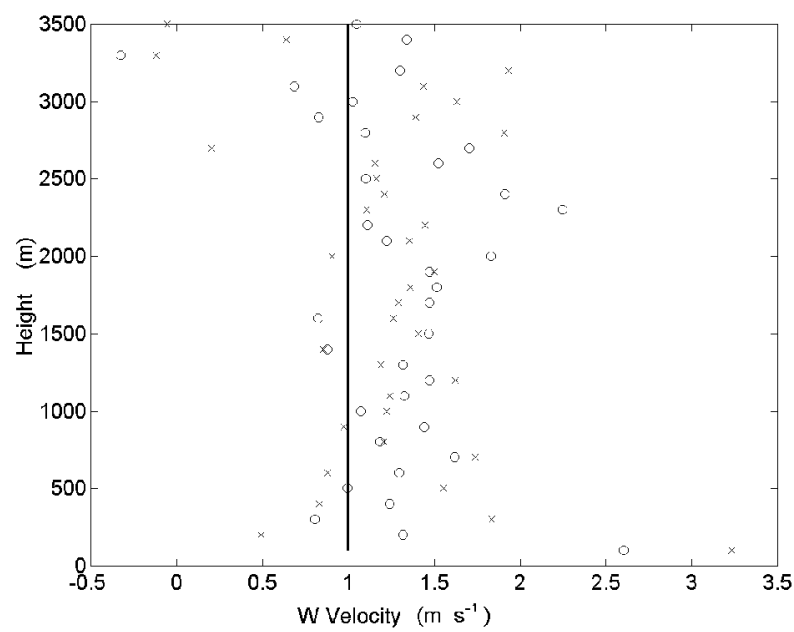

Figure 4. The line shows the mean $w$-wind profile input into the model; crosses $(\times)$ and circles (o) denote vertical wind velocities, derived from the idealized model, from positive and negative range data respectively.

The reason for this analysis of idealized data was twofold. Firstly, it gave an accurate estimate of the errors on the calculated velocity profiles produced using a leastsquares technique. The 'errors' on the model data are produced from the combination of the limited dataset, the added 'turbulence' and the analysis technique. Further errors associated with the real datasets are: missing data points, clutter points (points with too large returns) and horizontal inhomogeneities. These points will be discussed later.

The second reason for using an idealized dataset was to enable an investigation of how the size of the dataset affected the accuracy of the derived profiles. G-C92 comment that their $w$ profiles were derived from data in the range $30^{\circ}$ to $42^{\circ}$. It was found using the idealized data that reducing the number of points in the least-square technique to this level produced errors that were far larger than if the full range of elevation angles 
were used. It must be noted, however, that the derived $w$ values below the $500 \mathrm{~m}$ height were clearly unrealistic.

\section{(c) Retrieval errors}

As mentioned above, to calculate the most accurate measurement of wind velocities there is a trade off between a requirement for a large dataset to use for the statistical routine, and the need for each point used to be a good measurement of the wind velocity. It was found that by increasing the size of the dataset (i.e. using data from $0^{\circ}$ to $60^{\circ}$ for the calculation of both horizontal and vertical winds) the error on the derived wind velocity was reduced compared with that of the $0^{\circ}$ to $42^{\circ}$ dataset.

For the horizontal winds it can be seen from Fig. 3 that derived velocities are close to the input mean velocity up to $2500 \mathrm{~m}$. Calculating the average error on the derived horizontal winds, $u$, from $0-2500 \mathrm{~m}$ gives a standard deviation of $0.24 \mathrm{~m} \mathrm{~s}^{-1}$. Pearson and Collier (1999) note that the random error associated with an individual radial velocity estimate using the Salford lidar is $0.5 \mathrm{~m} \mathrm{~s}^{-1}$. G-C92 estimate that since each data point in their average scan is an average of more than 2000 observations then the error on each radial wind velocity, and hence the error on the horizontal wind velocities, is in the region of $1.5 \mathrm{~cm} \mathrm{~s}^{-1}$. This does not, however, account for the error inherent in the least-square-fit technique. This error is associated with how well the data fits to the $n$th order polynomial defined by Eq. (3) (where $n=1$ ), and Eq. (6) (where $n=2)$. The importance of analysing an idealized dataset to determine horizontal and vertical velocity errors can thus be seen.

The vertical velocity error was calculated using three datasets using different elevation angles; (1) $0^{\circ}$ to $60^{\circ}$, (2) $0^{\circ}$ to $45^{\circ}$ and (3) $30^{\circ}$ to $45^{\circ}$. For the first dataset, data from $500 \mathrm{~m}$ to $2500 \mathrm{~m}$ in height was deemed to have given good vertical velocity estimates (as shown in Fig. 4). The average vertical velocity was calculated to be $1.3 \mathrm{~m} \mathrm{~s}^{-1}$ with a standard deviation of $0.3 \mathrm{~m} \mathrm{~s}^{-1}$.

Using the second dataset useful estimates were deemed to be from $500 \mathrm{~m}$ to $1500 \mathrm{~m}$. An average over this height range again gave $w=1.3 \mathrm{~m} \mathrm{~s}^{-1}$ and a standard deviation of $0.3 \mathrm{~m} \mathrm{~s}^{-1}$. With the third dataset (which had a considerably reduced number of points) the average over $500 \mathrm{~m}$ to $1500 \mathrm{~m}$ gave a vertical velocity of $1.2 \mathrm{~m} \mathrm{~s}^{-1} \pm 1.4 \mathrm{~m} \mathrm{~s}^{-1}$. It can therefore be seen that in the retrieved data from the $30^{\circ}$ to $45^{\circ}$ dataset the vertical average estimate of the vertical component of the radial winds is more accurate, but the reduction of data points in the statistical routine produces much larger errors on the individual estimates in each height bin. The problem is that the least-square method is essentially looking at differences in the radial wind over a range of elevation angles to determine the vertical velocity. When the range of elevation angles is narrow then these differences are very small. If there are large amounts of data points within each height bin then the turbulence inherent in each radial velocity estimate is essentially averaged out, and the least-square-fit method can be used successfully. In G-C92 approximately 50 scans were carried out over a period of approximately an hour. In the data presented in this paper four scans were carried out over a period of approximately 20 minutes. With the data discussed in this paper it is then fair to say that there are insufficient data points over the $30-42^{\circ}$ range in elevation angles to determine accurately the vertical velocity. We have shown, however, that by taking the larger range in elevation angles we can estimate the vertical velocity to a similar accuracy.

In the estimates above there is a positive bias on the retrieved vertical velocity. To understand this, if we assume that the mean wind is purely horizontal, i.e. $w=0 \mathrm{~m} \mathrm{~s}^{-1}$, then the radial wind component will be $\alpha=u_{\mathrm{h}} \cos \Theta$. Where $u_{\mathrm{h}}$ is the horizontal wind 


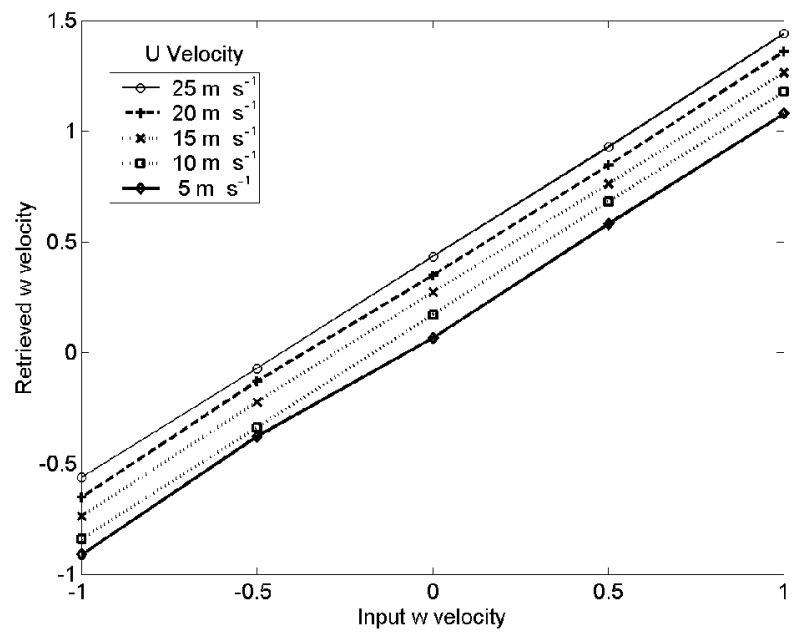

Figure 5. Series of curves to show the retrieved vertical velocity, $w$ (in $\mathrm{m} \mathrm{s}^{-1}$ ), given various input horizontal and vertical velocities.

velocity. In the method described above, the radial wind velocity is then split into vertical and horizontal components using Eq. (3). From geometrical considerations it is easy to see that the retrieved $w$ for any particular data point is bigger than zero. What we need to understand is how the positive bias on $w$ changes when we use our least-square method with various vertical and horizontal velocity profiles. To this end the model was run for horizontal wind velocity from 5 to $25 \mathrm{~m} \mathrm{~s}^{-1}$ and for vertical wind velocities from -1.0 to $1.0 \mathrm{~m} \mathrm{~s}^{-1}$. Figure 5 shows how the retrieved vertical velocity changes with changing input. It can be seen from Fig. 5 that the error on the vertical velocity is approximately a function of horizontal velocity $u$ only. For example, the error on the output $w$ is approximately a $+0.12 \mathrm{~m} \mathrm{~s}^{-1}$ bias for all values of $w$, when $u$ is set at $5 \mathrm{~m} \mathrm{~s}^{-1}$. When $u$ is set at $25 \mathrm{~m} \mathrm{~s}^{-1}$ the bias on $w$ is approximately $+0.43 \mathrm{~m} \mathrm{~s}^{-1}$ for all values of $w$. Since we can retrieve horizontal velocity data more accurately we can in future use this set of data to correct for the positive bias in $w$.

An error analysis was also carried out for the turbulence profiles, $\overline{u^{\prime 2}}, \overline{w^{\prime 2}}$, and $\overline{u^{\prime} w^{\prime}}$. Turbulence parameters were calculated by carrying out a least-square-fit analysis for the 2nd order polynomial defined in Eq. (6). Due to the large errors resulting from this analysis an extra step of statistical processing was also added. Since the least-square-fit technique was deemed to give a poor fit to the data points a method of resampling the data called the Bootstrap method (Wilks 1995) was used. In the Bootstrap method the actual dataset with its $2 \mathrm{~N}$ data points is used to generate any number of synthetic datasets, also with $2 N$ data points. The procedure is to resample the data with replacement. In this process, because of replacement of data, you get sets of data in which a random fraction of the original points, typically $1 / \mathrm{e} \approx 37 \%$, are replaced by duplicated original points. The technique is particularly good for this type of case where the results of the least-square fit can be unduly influenced by one or two bad data points. If the Bootstrap method is employed, however, the influence of bad data points is much reduced, and the Monte Carlo style simulations yield subsequent error estimates.

The results of this processing show that the turbulence profiles were approximately constant with height and the errors increase with height. Above $1500 \mathrm{~m}$ the errors are 
TABLE 1. INPUT TURBULENCE PARAMETERS AND THE RESUlTing OUTPUT TURBULENCE PARAMETERS RETRIEVED USING EQ. (6).

\begin{tabular}{lcc}
\hline & $\begin{array}{c}\text { Input turbulence } \\
\left(\mathrm{m}^{2} \mathrm{~s}^{-2}\right)\end{array}$ & $\begin{array}{c}\text { Output turbulence } \\
\left(\mathrm{m}^{2} \mathrm{~s}^{-2}\right)\end{array}$ \\
\hline Horizontal velocity variance $\overline{\left(\overline{u^{\prime 2}}\right)}$ & 0.29 & $0.38 \pm 0.29$ \\
Vertical velocity variance $\overline{\left(w^{\prime 2}\right)}$ & 0.14 & $0.02 \pm 0.68$ \\
Momentum flux $\left(\overline{u^{\prime} w^{\prime}}\right)$ & 0.00 & $0.18 \pm 0.51$ \\
\hline
\end{tabular}

Output turbulence parameters were averaged from $100 \mathrm{~m}$ to $1500 \mathrm{~m}$.

very large. For the $\overline{w^{\prime 2}}$ profile the errors also increase below $500 \mathrm{~m}$. Average profiles were calculated from $100 \mathrm{~m}$ to $1500 \mathrm{~m}$. The derived turbulence statistics are shown in Table 1 against the actual input turbulence. The results suggest that with spatially homogeneous turbulence this methodology does a good job of estimating the turbulence intensities.

Using conventional error analysis G-C92 estimate their momentum flux error to be $0.1 \mathrm{~m}^{2} \mathrm{~s}^{-2}$. Their theoretical estimate relies upon several assumptions mentioned previously: that the turbulence is horizontally homogeneous; that the observations are uncorrelated and nonbiased; that the datasets are large enough so that the least-square-fit analysis gives a good fit of the data; and, also, considering the nature of the least-squarefit analysis, that each and every data point is a good estimate of the radial wind velocity. By our use of the idealized model we see how well the methodology works in practice. In the idealized data the input turbulence components $u^{\prime}$ and $w^{\prime}$ were uncorrelated. The derived output turbulence, $0.18 \pm 0.51\left(\mathrm{~m}^{2} \mathrm{~s}^{-2}\right)$, was therefore a good estimate. The error of $0.51\left(\mathrm{~m}^{2} \mathrm{~s}^{-2}\right)$ was, however, larger than theoretically estimated by G-C 92 . It was our use of the Bootstrap method which gave us our errors. Essentially, by subsampling the data we get slightly different values for the turbulence parameters. These values give us our error bars. G-C92 note that when they sub-sampled their data their values were 'quite close', but do not comment further. The fact that our error bars are larger than those of G-C92 is therefore a result of the statistical nature of the leastsquare-fit routine which G-C92 do not consider in their theoretical analysis.

\section{OBSERVATIONS}

\section{(a) Synoptic situation}

The field trial was conducted on 13 March 2000. The weather was characterized by a high-pressure system which had been stationary over the British Isles for approximately 4 days before the trial. There was $8 / 8$ cloud cover over the site. A radiosonde ascent from Aberporth taken at 17:00 UTC showed the atmosphere was conditionally unstable. The level of free convection was calculated to be approximately $1400 \mathrm{~m}$, with a possible layer of stratus cloud at $900 \mathrm{~m}$. The wind direction from the radiosonde ascent showed the surface winds to be south-westerly $\left(220^{\circ}\right)$ turning to north-westerly $\left(300^{\circ}\right)$ above $600 \mathrm{~m}$.

The centre of the field site was approximately $2 \mathrm{~km}$ east of the peak of the Malvern hills. The Malvern hills are a $6 \mathrm{~km}$ long ridge of peaks aligned in a north-south direction. The height of the ridge is between $350 \mathrm{~m}$ and $450 \mathrm{~m}$ above sea level and its half-height width is between $0.5 \mathrm{~km}$ and $1.0 \mathrm{~km}$. The field site was approximately $60 \mathrm{~m}$ above sea level. Data were taken over a period of approximately one hour starting at 16:00 UTC. Firstly, a set of data was taken which consisted of four $180^{\circ}$ RHI scans perpendicular to 


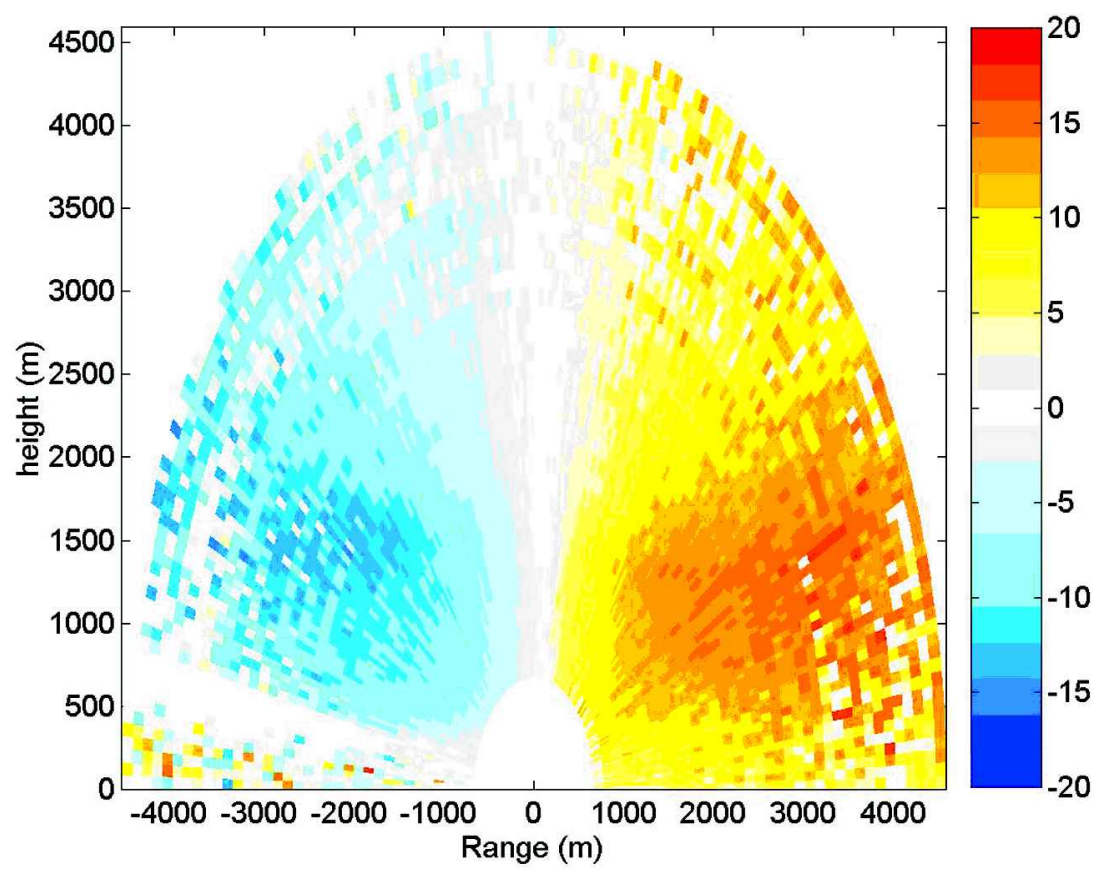

Figure 6. Lidar radial wind velocity scan averaged over a 20-minute period at approximately 16:00 UTC 13 March 2000. (Colour bar shows wind velocity in $\mathrm{m} \mathrm{s}^{-1}$.)

the Malvern hills (the $x-z$ plane). These were taken from 16:00 to 16:20 UTC. A second set of four RHI scans was taken parallel to the hills (i.e. in the $y-z$ plane) from 16:20 to $16: 40 \mathrm{UTC}$, but this paper does not cover the analysis of this data. Thirdly, a $360^{\circ}$ azimuth scan was done at an elevation of $45^{\circ}$, from 16:40 to 17:00 UTC.

\section{(b) Doppler lidar wind profiles}

The first step in data analysis was to filter the raw radial velocity returns. Two filtering steps were taken. Firstly, all data points where the signal intensity was below a certain threshold were eliminated. Where the backscattered signal intensity is below the system noise level the processing produces bad Doppler velocity returns and these must be removed. Secondly, all points where the velocity returns were above $23 \mathrm{~m} \mathrm{~s}^{-1}$ were also eliminated. The derived wind velocities are not sensitive to this cut-off and using thresholds of $20 \mathrm{~m} \mathrm{~s}^{-1}$ or $26 \mathrm{~m} \mathrm{~s}^{-1}$ both produced very similar results. This filtering removes the unrealistic returns that are due to 'clutter', e.g. birds and insects. Then each set of four RHI scans was averaged to produce one average scan. The average scan in the $x-z$ plane is shown in Fig. 6 . The lowest $20^{\circ}$ on the left-hand side of the scan shows no sensible data due to the presence of the hills.

Using the G-C92 method, data were then separated into height 'bins' spaced at $100 \mathrm{~m}$ intervals. Then the least-square-fit analysis was carried out to derive the $u$ and $w$ wind velocities. The derived $u$ and $w$ velocities are shown in Figs. 7 and 8 respectively. Because of the presence of the ridge, and since the wind was primarily in a westerly direction, it was considered that the profiles in the upstream and downstream directions (relative to the lidar position) could possibly have very different characteristics. It was 


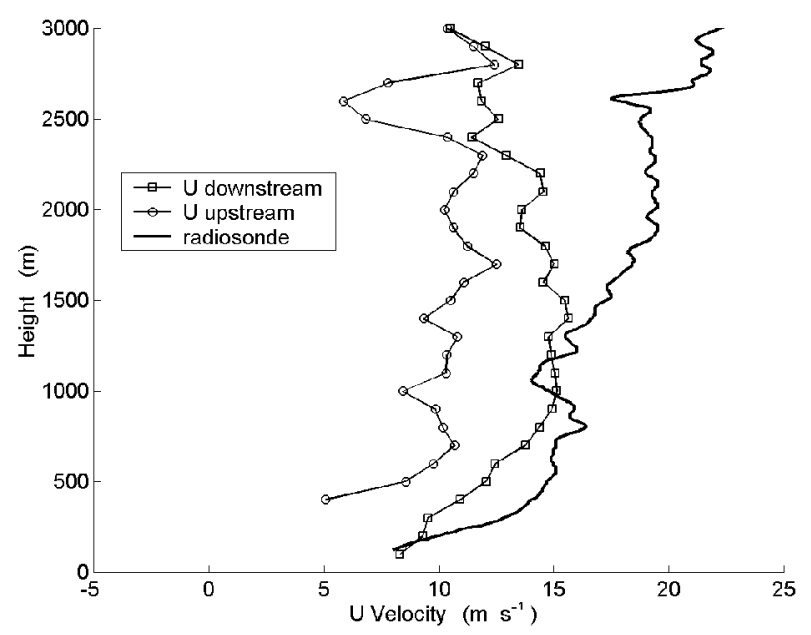

Figure 7. Horizontal wind profile derived from lidar data showing profiles upstream and downstream of the lidar separately. The 17:00 UTC radiosonde data from Aberporth is also shown.

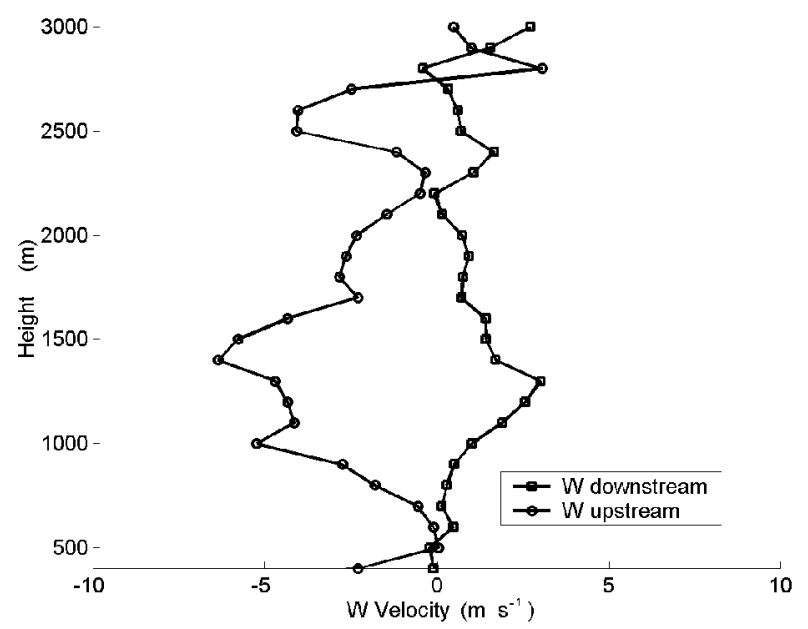

Figure 8. Vertical wind profile derived from lidar data showing profiles upstream and downstream of the lidar separately.

therefore deemed sensible to analyse data in the upstream and downstream directions separately.

Data for the upstream section shows descent and a reduction in horizontal wind velocity. The derived profiles from this section of data are wind velocities that have been averaged over highly changing terrain. The assumption of horizontal homogeneity used in the processing of the data is not a good assumption in this case. If the lidar was primarily looking at data in the wake region of the hill then a reduction in $u$ would be expected. Using temperature data from the Aberporth radiosonde ascent, the static stability can be calculated. This can be used to calculate the Froude number, Fr, which is a ratio of the atmospheric natural oscillation wavelength to the effective wavelength of the hill (Stull 1988). For this case the Froude number was estimated to be $F r=1.5-$ 4.5. Flow over a hill, with Froude numbers in this region, would be expected to produce 


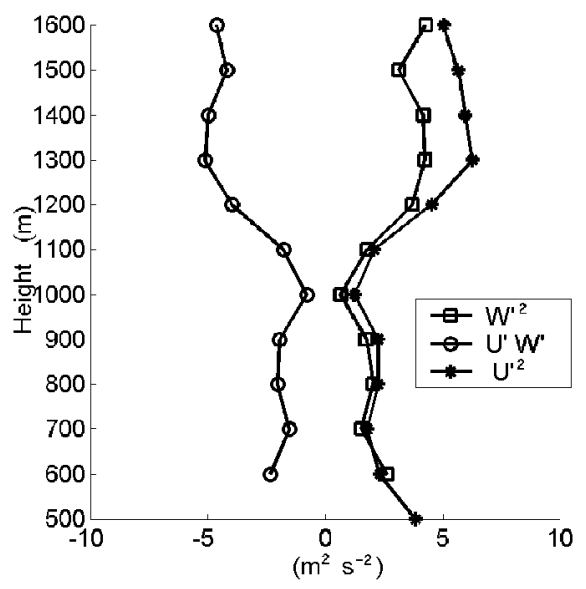

Figure 9. Derived turbulent fluxes; horizontal velocity variance $\left(\overline{u^{\prime 2}}\right)$, vertical velocity variance $\left(\overline{w^{\prime 2}}\right)$, and momentum flux $\left(\overline{u^{\prime} w^{\prime}}\right)$, downstream of the lidar position.

boundary-layer separation of the flow and a turbulent wake (Stull 1988). Two minima are shown in the vertical velocity profile which coincide with the predicted cloud base and stratus levels.

In the downstream data the horizontal wind flow is comparable with that from the radiosonde (Fig. 7) in the lowest $1400 \mathrm{~m}$. In the radiosonde profile the wind increases from $8 \mathrm{~m} \mathrm{~s}^{-1}$ at $100 \mathrm{~m}$ to approximately $16 \mathrm{~m} \mathrm{~s}^{-1}$ at $1000 \mathrm{~m}$. The vertical velocity in the downstream case has a maxima at $1300 \mathrm{~m}$.

Calculation of the turbulence parameters showed marked differences in the upstream and downstream cases. In the upstream data (i.e. the data over the brow of the hill) all parameters show very large values. This is assumed to be due to the invalid assumption of horizontal homogeneity and these turbulence profiles are not discussed further.

In the downstream data all the turbulence parameters showed large errors below $500 \mathrm{~m}$ and above $1600 \mathrm{~m}$. Figure 9 shows the derived horizontal velocity variance, $\left(\overline{u^{\prime 2}}\right)$, the derived vertical velocity variance, $\left(\overline{w^{\prime 2}}\right)$, and the derived momentum flux, $\left(\overline{u^{\prime} w^{\prime}}\right)$. These profiles show a reduction in the turbulence away from the surface. The momentum flux is negative, and momentum is therefore being transferred downwards by the eddies. The horizontal wind velocity increases with height (Fig. 7), and the sign of the momentum flux in the lowest levels is therefore in agreement with eddy viscosity theory (Panofsky and Dutton 1984).

From $700 \mathrm{~m}$ to $1000 \mathrm{~m}$, within the stable layer, the turbulence profiles are approximately constant with height. They then show a large increase around the cloud-base level. G-C92 also find very large (negative) momentum flux values at the border of the mixed layer, which is in agreement with our values. Given that the turbulence due to the updraughts and downdraughts in the lowest layers of cloud structure must be very inhomogeneous over even small distances (100 to $200 \mathrm{~m}$ ), it is not surprising that these values of momentum flux are much larger than those quoted by other researchers in non-convective conditions (Eberhard et al. 1989). Price (2000) discusses the structure of a cloudy boundary layer and shows momentum fluxes, averaged over approximately a 1-hour period, of $-0.2 \mathrm{~m}^{2} \mathrm{~s}^{-2}$ near to the ground and $-0.15 \mathrm{~m}^{2} \mathrm{~s}^{-2}$ near the top of 
the cloud layer, but note that between these levels the data were of insufficient data quality'. The values of the turbulence parameters are inline with those of G-C92, who are the only group making similar spatially averaged measurements.

G-C92 compare their retrieved vertical velocity with that obtained by the beam as it is pointed vertically. These two profiles are very different in their structure. They subsequently use the vertical velocity that has been calculated from the vertical beam only to calculate their turbulence profiles. The vertical data were not used in this study as data upstream of the lidar and downstream of the lidar were analysed separately, and a vertical velocity directly over the lidar position was considered not to be characteristic of either dataset.

\section{DISCUSSION AND CONCLUDING REMARKS}

Analysis of radial velocity data retrieved from a single Doppler lidar has been carried out using a method previously documented by G-C92. Error analysis of the methodology has been done by employing a simple idealized scan to simulate a known velocity and turbulence profile. Using an idealized dataset we could calculate the errors on the derived quantities given 'perfect' and known input data. In real data there are always problems associated with bad data points and inhomogeneities as well as the problem of actually not knowing the 'real' answer. Using this model data we can answer the question of how well the processing technique works to predict the answer.

By the use of this simple idealized input we showed that the derived profiles reproduce successfully the input wind and turbulence profiles. The error analysis shows that wind velocity profiles can be derived to an accuracy of $0.24 \mathrm{~m} \mathrm{~s}^{-1}$ in the horizontal and $0.3 \mathrm{~m} \mathrm{~s}^{-1}$ in the vertical. This is in the region of a $2 \%$ error in the horizontal wind and $30 \%$ error in the vertical wind. G-C92 estimated that the error on the derived horizontal wind velocity was of the order of $1.5 \mathrm{~cm} \mathrm{~s}^{-1}$. Given the error analysis with our idealized input data the least-square routine clearly gives much larger errors than they predicted.

The derived vertical velocity was found to have a positive bias. This was shown to be approximately dependent on the horizontal wind velocity only and can therefore be corrected for. To understand the effect of having a more limited dataset the vertical velocity was calculated from reduced datasets. G-C92 used only data from $30^{\circ}$ to $42^{\circ}$ in their analysis. We found using only data from these angles that the error on the vertical velocity estimate for each analysis height was much larger.

Using the idealized data, turbulence profiles were also calculated. These showed that theoretically, given ideal data, turbulence profiles were derived to an accuracy of $0.29 \mathrm{~m}^{2} \mathrm{~s}^{-2}$ for $\overline{u^{\prime 2}}, 0.68 \mathrm{~m}^{2} \mathrm{~s}^{-2}$ for $\overline{w^{\prime 2}}$, and $0.51 \mathrm{~m}^{2} \mathrm{~s}^{-2}$ for $\overline{u^{\prime} w^{\prime}}$. The wind velocities were accurate to a height of $2500 \mathrm{~m}$, whereas the turbulence parameters were only accurate to a height of $1500 \mathrm{~m}$.

The DERA Malvern lidar was deployed to examine the flow over the Malvern hills on a day in which the surface layer was stable. Data in the upwind and downwind positions were analysed separately. This was due to the fact that data upwind of the lidar was effectively data taken over the brow and in the lee of the hill. Data taken in the downwind direction was effectively taken from $2 \mathrm{~km}$ to $5 \mathrm{~km}$ downstream of the hill.

Profiles downwind of the lidar position were calculated over flat terrain. The downwind $u$ showed an increase from $8 \mathrm{~m} \mathrm{~s}^{-1}$ at $100 \mathrm{~m}$ to $15 \mathrm{~m} \mathrm{~s}^{-1}$ at $1000 \mathrm{~m}$. This was consistent with the radiosonde ascent. Above $1000 \mathrm{~m} u$ was approximately constant, until slightly decreasing above $2100 \mathrm{~m}$. The $w$ profile showed a positive maximum at a 
height of $1300 \mathrm{~m}$. This was thought to be possibly due to lee-wave activity, which would fit with cloud-band structure seen in satellite data (not shown) for the day in question.

The atmospheric data analysed in this work was clearly not ideal in that they cannot be considered to be homogeneous, as required by the processing technique. Unfortunately atmospheric conditions are seldom homogeneous and for our purposes we must then ask the question 'do we gain any understanding of the vertical structure of the atmosphere by using this technique even in these types of conditions?'.

It is quite clear that one might possibly want to know the spatially averaged mean wind profiles over a large area. However, it is not quite so clear what we are measuring when we calculate the turbulence parameters. By averaging the data initially over a time period of 20 minutes (as we have done here for each scan), the aim is to produce a more statistically stable dataset; i.e. to average out bad or suspect data points. The turbulence calculated from this time-averaged data is then a measure of how the data vary spatially. With point sensors we have to evoke Taylors 'frozen' turbulence hypothesis to translate the measurements as a function of time to their corresponding measurements in space. There is of course no guarantee with point sensors that the turbulence at the location of the sensor is characteristic of turbulence over an area surrounding the sensor. With the lidar data we are actually measuring the area-averaged turbulence over the lidar domain. What we do have to be aware of is that the domain of averaging is to some extent predetermined.

For the data in the downwind case the horizontal wind velocity profile was comparable to the radiosonde data. The turbulence profiles for the downwind case were large near the lowest resolved levels and decreased in magnitude up to the approximate cloud-base height $(1000 \mathrm{~m})$. Above this they increased in magnitude to a maximum at $1300 \mathrm{~m}$. With this turbulence data we do have to consider the consequences of the possible lee-wave behaviour mentioned above. Calculations from the available data estimate the possible lee-wave wavelength to be in the region of $15 \mathrm{~km}$. Over the distance of the downwind lidar data, $2-5.5 \mathrm{~km}$ downwind of the hill, the presence of a lee wave would be evident over this relatively short distance as a trend in the mean wind profiles. Therefore the turbulence as calculated from this dataset would contain some element of this trend. Further work needs to be done to enhance this analysis technique to remove any possible trend from the data, but in the analysis in this paper this has not been done. The sign and magnitude of the fluxes calculated in this paper are, however, in agreement with those of G-C92 which were calculated over more homogeneous orography.

In the case of the upstream profiles the condition of horizontal homogeneity was obviously broken. The wind velocity profiles show descent in the lee of the hill and a slow down in $u$ possibly due to a wake region, this could not be confirmed. The turbulence profiles could not be calculated.

As with other meteorological field experiments it would have given a clearer picture if we had had more direct measurements with which to compare. Unfortunately there are very few real measurements of the boundary-layer flow up to the heights discussed in this paper. The challenge with Doppler lidar data, as with any new instrument, is how to interpret the available data in a useable context. This paper has demonstrated that a single pulsed Doppler lidar has the capabilities to produce velocity and turbulence profiles which are theoretically accurate up to a level of $2500 \mathrm{~m}$ and $1500 \mathrm{~m}$ respectively. The possibility of having observations of turbulence parameters throughout the whole depth of the boundary layer has the ability to aid our understanding of the structure of the boundary layer under many different meteorological conditions.

It must also be noted that it is difficult to compare the profiles that are derived using this method with those of point measurements because they are effectively 
area-averaged profiles. However, it could be said that these area-averaged profiles are more useful than point measurements as they can be more easily compared with the larger-scale output derived from computer models.

\section{ACKNOWLEDGEMENTS}

Thanks are due to the technical staff at the University of Salford and QinetiQ involved in the lidar research. The work was partly funded under the Natural Environment Research Council URGENT Research grant GST/02/2231.

Browning, K. A. and Wexler, R.

Eberhard, W. L., Cupp, R. E. and Healy, K. R.

Gal-Chen, T., Xu, M. and Eberhard, W. L.

Kuettner, J. P., Hildebrand, P. A. and Clark, T. L.

Kunkel, K. E., Eloranta, E. W. and Weinman, J. A.

Panofsky, H. A. and Dutton, J. A.

Pearson, G. N. and Collier, C. G.

Price, J. D.

Stull, R. B.

Wilks, D. S.

Wilson, D. A.

\section{REFERENCES}

1968 The determination of kinematic properties of a wind field using a Doppler radar. J. Appl. Meteorol., 7, 105-113

1989 Doppler lidar measurement of profiles of turblence and momentum flux. J. Atmos. Oceanic Technol., 6, 809-819

1992 Estimations of atmospheric boundary layer fluxes and other turbulence parameters from Doppler lidar data. J. Geophys. Res., 97, 18409-18423

Convection waves: Observations of gravity wave systems over convectively active boundary layers. Q. J. R. Meteorol. Soc., 113, 445-467

1980 Remote determination of winds, turbulence spectra and energy dissipation rates in the boundary layer from lidar measurements. J. Atmos. Sci., 37, 978-985

1984 Atmospheric turbulence: Models and methods for engineering applications. Wiley

1999 A compact pulsed coherent $\mathrm{CO}_{2}$ laser radar for boundary-layer meteorology. Q. J. R. Meteorol. Soc., 125, 2703-2721

A case-study of a partially cloudy boundary layer. $Q . J . R$. Meteorol. Soc., 126, 393-413

1988 An introduction to boundary-layer meteorology. Kluwer Academic Publishers

1995 Statistical methods in the atmospheric sciences. Academic Press

1970 'Doppler radar studies of boundary layer wind profile and turbulence in snow conditions'. Pp. 191-196 in Proceedings of the 14th radar meteorology conference, Tucson, USA. American Meteorological Society, Boston, USA 